\title{
Dimensional Changes of Polypeptides in the Helix-Sense Inversion Region
}

\author{
Hirokazu TORIUMI \\ Department of Chemistry, College of Arts and Sciences, \\ University of Tokyo, 3-8-1 Komaba, Meguro, \\ Tokyo 153, Japan
}

(Received July 25, 1987)

\begin{abstract}
Dimensional changes of polypeptides in the helix-sense inversion region are analyzed by a theory based on the three-state, nearest-neighbor interaction model. Each peptide residue in a chain is assumed to take on right- and left-handed $\alpha$-helical states as well as a random coil state, and the helix stabilization effect due to hydrogen-bond interactions is taken into account. Such interactions between four consecutive residues have explicitly been discussed in a $9 \times 9$ statistical-weight matrix in a previous theory [Macromolecules, 17, 1599(1984)], while in the present model we simplify this matrix into the form of a $3 \times 3$ matrix by considering the interactions only between two consecutive residues. Calculations of the average helix fraction and the number of helix sequences are correspondingly simplified with maintaining quantitative agreement in numerical results. The new theory is also applied to the analyses of the length distribution and the higher moments of helix. Theoretical transition curves illustrating the general characteristics of the sense inversion phenomena suggest a method for evaluating the transition parameters.

KEY WORDS Polypeptide / Helix-Sense Inversion / Chain Dimension / Nearest-Neighbor Interaction Model/
\end{abstract}

In a previous study ${ }^{1}$ we have developed a theory of the helix-sense inversion in polypeptides for interpreting the unusual conformational transitions exhibited by poly(aspartate ester)s. For example, $\operatorname{poly}(\beta$ phenethyl L-aspartate) (PPELA), being in a right-handed $\alpha$-helical form (RH $\alpha$ ) in chloroform, undergoes a sense inversion to a lefthanded $\alpha$ form ( $\mathrm{LH} \alpha$ ) when a small amount of denaturing solvent such as dichloroacetic acid or trifluoroacetic acid is added. ${ }^{2^{\prime}}$ The LH $\alpha$ helix thus formed can then be transformed into a random coil form by further increasing the acid concentration. Moreover, PPELA in an appropriate chloroform-acid mixture undergoes a thermally induced sense inversion from $\mathrm{RH}$ to $\mathrm{LH} \alpha$-helix upon heating. An indication of this latter type of inversion has also been reported for poly $(\beta-n$-propyl L-aspartate) in chloroform. ${ }^{3.4}$ Generally, the stabilities of the two $\alpha$-helical forms of poly(aspartate ester)s are known to be low and close together. ${ }^{3-8}$ The sense inversion can therefore be induced relatively easily in this class of polypeptides when the solution conditions are appropriately changed.

In the theory in ref 1 (hereafter referred to as Part I) we have assumed that each peptide residue in a chain can take on three conformational states ( $\mathrm{RH} \alpha, \mathrm{LH} \alpha$ and coil states), and that the conformational stability of a given residue depends on the states of its neighboring three residues. This latter requirement arises since a hydrogen-bond bridge stabilizing the helix sequence forms only when three consecutive residues are cooperatively distorted to the $\alpha$-helical state of the same sense. A statistical-weight matrix representing this long-range hydrogen-bond interaction is given by a $9 \times 9$ matrix with five non-zero 
eigenvalues. The partition function of the entire chain is derived in terms of these five eigenvalues and used to obtain explicit expressions for average fractions of the two $\alpha$ helical conformers. The theory in Part $I$ is successful in reproducing the fundamental aspects of the sense inversion phenomena; nevertheless, its scope and applicability are expected to be further expanded before we proceed to the analyses of experimental observations.

Particularly, the following problems are to be noted. In most current experiments, conformational properties of polypeptides are examined by polarimetric measurements such as optical rotatory dispersion (ORD) and circular dichroism (CD). In the case of a simple helixcoil transition both ORD and $\mathrm{CD}$ can readily determine an average helix fraction: However, when the chain undergoes the sense inversion, individual values of fractions of $\mathrm{RH}$ and $\mathrm{LH} \alpha$ conformers can no longer be separated. The quantity measurable in these experiments is a fractional difference between the two $\alpha$-helical conformers $\left(f_{\mathrm{r}}-f_{1}\right)$; while the sum of their fractions $\left(f_{\mathrm{r}}+f_{1}\right)$, i.e., a total helicity, is left undetermined. Estimation of the latter quantity should require independent experimental informations (e.g., the mean-square radius of gyration, the dipole moment, or the Kerr constant from electric birefringence measurement) that reflect the average dimension of $\alpha$ helices irrespective of their right- or lefthandednesses. It is for this reason that we have carried out in a recent study ${ }^{9}$ the measurement of electric birefringence of poly $(\varepsilon$-carbobenzoxy L-lysine) (PCBL) in the helix-coil transition region. This particular polypeptide is chosen since it undergoes a sharp transition and detailed conformational properties have already been established through several experimental studies. ${ }^{10-13}$ The observed changes in birefringence can well be reproduced from ORD data by using the Zimm-Bragg theory ${ }^{14}$ where only the nearest-neighbor interactions between two consecutive residues are considered. This reference experiment suggests that the informations needed to analyze the sense inversion mechanism can similarly be obtained from electric birefringence measurements (and probably from other two experiments as well), and that the nearest-neighbor interaction model should work in these analyses. This second conclusion is important since all aforementioned studies require theoretical estimation of the higher order moments of helix for analyzing the observed informations (e.g., the electric birefringence, or the Kerr constant, is in proportion to an average of the cubed helix lengths). ${ }^{9}$ To facilitate these calculations and hence provide a means of quantitative analyses of experimental observations, we simplify below the theory in Part I by eliminating mathematical complexity associated with the long-range hydrogen-bond interactions.

The nearest-neighbor interaction model including three conformational states was once discussed by Scott. ${ }^{15}$ His primary interest was to see how the $\alpha$-helical conformation of one sense can be stabilized in a long polypeptide chain in spite that the energy difference between $\mathrm{RH}$ and $\mathrm{LH} \alpha$ states of each residue has been predicted to be only marginal. ${ }^{16.17}$ Although he was aware of possibility of coexistence of RH and $\mathrm{LH} \alpha$-helices in the intermediate stages of the helix-coil transition, no further attention was paid to the sense inversion phenomena. In the following we analyze the dimensional changes of a polypeptide chain in the sense inversion region based on this nearest-neighbor interaction model, and propose a method for estimating the thermodynamic parameters of the transition.

\section{THEORY}

\section{Nearest-Neighbor Interaction Model}

Some basic assumptions introduced in Part I to describe the sense inversion phenomena are as follows. A polypeptide chain under consideration is regarded to consist of a linear 
array of monomeric residues $\left(-\mathrm{CO}-\mathrm{C}^{\alpha} \mathrm{HR}-\right.$ $\mathrm{NH}-$ ), with $\mathrm{R}$ being a side chain attached to the $\alpha$-carbon atom. All monomeric residues are indexed as $1,2, \cdots, N$ from the free carboxyl end, so that an intramolecular hydrogen-bond bridge forms between the amido hydrogen atom of a given residue (say, $i-2$ ) and the amido oxygen atom of its fourth succeeding residue $(i+2)$ when intervening three residues $(i-1, i$, and $i+1)$ are all distorted to the $\alpha$-helical state. Such a residue, distorted to the $\alpha$-helical state and stabilized by at least one hydrogen-bond bridge, is defined as a helix (h) unit. Since each individual residue is assumed to interconvert its conformational state between $\mathrm{RH}$ and $\mathrm{LH} \alpha$-helical states, the $\mathrm{h}$ units are distinguished into two types. One which has the rotational angles about the skeletal single bonds $\mathrm{C}-\mathrm{C}^{\alpha}$ and $\mathrm{C}^{\alpha}-\mathrm{N}$ equal to those characteristic of the $\mathrm{RH} \alpha$ conformation is defined as a $r$ unit, and the other one having the rotational angles equal to those of the $\mathrm{LH}$ $\alpha$ conformation is defined as a 1 unit. A residue assuming any arbitrary sets of angles other than the above two is then called a random coil unit and denoted by $\mathrm{c}$.

According to the requirement that the helix sequence becomes stabilized when it involves three consecutive helix units, occurrences of che and chhe $(\mathrm{h}=\mathrm{r}$ or $\mathrm{l})$ sequences with only one or two helix units are disregarded. ${ }^{18.19}$ This latter tetrad chhc is the longest sequence whose interaction is to be considered, hence a statistical-weight matrix (or a transition matrix) assumed in Part I has originally had a dimension of $3^{3} \times 3^{3}$. Fortunately, some of these 27 triads have been excluded by considering the steric hindrance effects between $r$ and 1 units and the hydrogen-bond interactions. The dimension of this matrix has finally been reduced to $9 \times 9$ after eliminating redundant matrix elements.

In the nearest-neighbor interaction model adopted here, we disregard some detailed structural characteristics of the hydrogenbond bridge and instead introduce a hypo- thetical bridge. That is, when a given residue (i) turns into an $\alpha$-helical state, a hypothetical hydrogen-bond bridge forms with its neighboring residue $(i-1)$ and thus stabilizes the helical conformation of the $i$ th residue. A statistical-weight matrix $\boldsymbol{W}$ sufficient to describe this situation may have the following structure $^{15}$

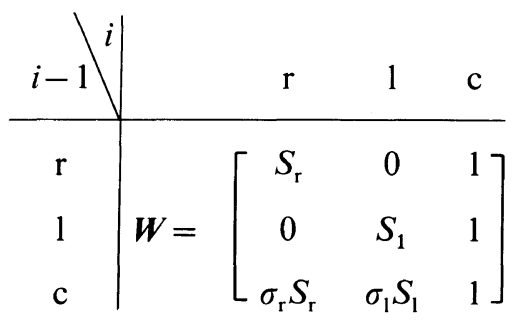

where $S_{\mathrm{h}}$ and $\sigma_{\mathrm{h}}(\mathrm{h}=\mathrm{r}, 1)$ represent an equilibrium constant and a cooperative parameter of the helix formation, respectively. Fundamentals of our three-state, nearestneighbor interaction model are selfexplanatory in eq 1 . The $(1,1)$ and $(2,2)$ elements of $\boldsymbol{W}$ having the statistical weight $S_{\mathrm{h}}$ characterize the helix propagation process maintaining the same sense, while the $(3,1)$ and $(3,2)$ elements with $\sigma_{\mathrm{h}} S_{\mathrm{h}}$ characterize the helix initiation process from coil residues. In the third column the same statistical weight of unity is assigned to all coil residues irrespective of the conformational state of their preceding residues. The $(1,2)$ and $(2,1)$ elements of $\boldsymbol{W}$, whose statistical weights are taken to be zero, prohibit the successive occurrence of $r$ and 1 units ( $c f$. Part I). Hence, in the matrix $W$ in eq 1 , the sense inversion is expressed in terms of the coexistence of two equilibria; $\mathrm{RH} \alpha \rightleftharpoons$ coil and coil $\rightleftharpoons \mathrm{LH} \alpha$. In other words, the inversion is assumed to proceed with retaining at least one coil residue between $\mathrm{RH}$ and $\mathrm{LH} \alpha$-helices.

The matrix contraction by the nearestneighbor interaction model may require certain adjustments of the elements. In a real polypeptide chain, the growth of helix sequence may continue from either end of the sequence with an equal probability. The $9 \times 9$ transition matrix in Part I was large enough to 
distinguish the first and last residues in the sequence, hence we were able to represent this two-directional growth of helix by assigning the same statistical weight $\sqrt{\sigma_{\mathrm{h}}}$ to these two terminal residues. However, in the contracted $3 \times 3$ matrix $\boldsymbol{W}$ in eq 1 we cannot designate the last helix residue in the sequence since every $h$ unit, except the first one in the sequence, is given the same statistical weight $S_{\mathrm{h}}$ no matter whether it is followed by h or c. Consequently, the $\sqrt{\sigma_{\mathrm{h}}}$ value assumed in Part I for the last helix residue is not counted in the present matrix $\boldsymbol{W}$. To compensate this we assign a statistical weight of the order of $\sigma_{\mathrm{h}}$ to the $(3,1)$ and $(3,2)$ elements of $\boldsymbol{W}$ which are responsible for the helix initiation process. Then, for the purpose of simplicity, we replace this $\sigma_{\mathrm{h}}$ value by $\sigma_{\mathrm{h}} S_{\mathrm{h}}$ and let all matrix elements in the first and second columns include $S_{\mathrm{h}}$ in common. It should be clear that our $S_{\mathrm{h}}$ and $\sigma_{\mathrm{h}}$ parameters have the same physical meanings as those discussed in the Zimm-Bragg theory of the helix-coil transition. ${ }^{14.20}$ As will be seen below, mathematical calculations of various molecular averages can be simplified by this modification without causing any significant influences to the final numerical results (note that $S_{\mathrm{h}} \simeq 1$, hence $\sigma_{\mathrm{h}} S_{\mathrm{h}} \simeq \sigma_{\mathrm{h}}$, in the region of our interest).

\section{Partition Function}

The partition function of a polypeptide chain consisting of $N$ monomeric residues is given as

$$
Z_{N}=e_{1} W^{N-2} e_{N}
$$

The end vectors associated with the first and $N$ th residues, which are assumed never to be involved in the helix section, should have the form

$$
e_{1}=(0,0,1)
$$

and

$$
\boldsymbol{e}_{N}=(1,1,1)^{\mathrm{T}}
$$

where the superscript $\mathrm{T}$ denotes the transpose of a vector. To evaluate $Z_{N}$, the matrix $W$ may be expanded by its eigenvalues and eigenvectors. The secular equation of $\boldsymbol{W}$ is given as

$$
\begin{aligned}
& \left(\lambda-S_{\mathrm{r}}\right)\left(\lambda-S_{1}\right)(\lambda-1) \\
& \quad-\sigma_{\mathrm{r}} S_{\mathrm{r}}\left(\lambda-S_{1}\right)-\sigma_{1} S_{1}\left(\lambda-S_{\mathrm{r}}\right)=0
\end{aligned}
$$

and its three roots, i.e., the eigenvalues of $\boldsymbol{W}$, are denoted by $\lambda_{i}(i=1-3)$ in the order of magnitude. The right-hand side and left-hand side eigenvectors of $\boldsymbol{W}$, denoted respectively by $\boldsymbol{u}(\lambda)$ and $\boldsymbol{v}(\lambda)$ for $\lambda=\lambda_{1}, \lambda_{2}$, and $\lambda_{3}$, can then be calculated as follows in the normalized form

$$
u(\lambda)=\left(1, \frac{\lambda-S_{\mathrm{r}}}{\lambda-S_{1}}, \lambda-S_{\mathrm{r}}\right)^{\mathrm{T}}
$$

and

$$
v(\lambda)=C(\lambda)\left(1, \frac{\sigma_{1} S_{1}}{\sigma_{\mathrm{r}} S_{\mathrm{r}}} \frac{\lambda-S_{\mathrm{r}}}{\lambda-S_{1}}, \frac{\lambda-S_{\mathrm{r}}}{\sigma_{\mathrm{r}} S_{\mathrm{r}}}\right)
$$

where

$$
\begin{gathered}
C(\lambda)=\sigma_{\mathrm{r}} S_{\mathrm{r}} \frac{\lambda-S_{1}}{\lambda-S_{\mathrm{r}}} F(\lambda) \\
F(\lambda)=\left\{\left(\lambda-S_{\mathrm{r}}\right)\left(\lambda-S_{1}\right)+A_{\mathrm{r}}(\lambda)+A_{1}(\lambda)\right\}^{-1} \\
A_{\mathrm{r}}(\lambda)=\left(\lambda-S_{\mathrm{r}}\right)(\lambda-1)-\sigma_{\mathrm{r}} S_{\mathrm{r}} \\
A_{1}(\lambda)=\left(\lambda-S_{1}\right)(\lambda-1)-\sigma_{1} S_{1}
\end{gathered}
$$

By combining eq $6-11$, we expand $\boldsymbol{W}$ as

$$
\boldsymbol{W}=\sum_{\lambda} \lambda \boldsymbol{u}(\lambda) v(\lambda)
$$

and hence

$$
\boldsymbol{W}^{N-2}=\sum_{\lambda} \lambda^{N-2} \boldsymbol{u}(\lambda) v(\lambda)
$$

Introduction of eq 13 to 2 gives the final expression of the partition function $Z_{N}$ :

$$
\begin{aligned}
Z_{N} & =\sum_{\lambda} \lambda^{N-2} e_{1} u(\lambda) v(\lambda) e_{N} \\
& =\sum_{\lambda} \lambda^{N-1}\left(\lambda-S_{\mathrm{r}}\right)\left(\lambda-S_{1}\right) F(\lambda)
\end{aligned}
$$

Average Dimension of $R H$ and $L H \alpha$-Helices

In the matrix $\boldsymbol{W}$ in eq 1 , occurrence of the $\mathrm{RH} \alpha$ state at the $i$ th residue is represented by the two different joint conformations, cr and 
rr. The former characterizes the $r$ residue appearing at the beginning of the $\mathrm{RH} \alpha$ sequence, and the latter characterizes that located either within or at the end of the sequence. Since both joint conformers have $S_{\mathrm{r}}$ in their statistical weights in common, the average number of $\mathrm{RH} \alpha$ residues per chain is given by the exponent of $S_{\mathrm{r}}$ in $Z_{N}$ :

$$
\begin{aligned}
N_{S_{\mathrm{r}}}= & \frac{\partial \ln Z_{N}}{\partial \ln S_{\mathrm{r}}} \\
= & \frac{1}{Z_{N}} \sum_{\lambda} \lambda^{N-1}\left\{N\left(\lambda-S_{\mathrm{r}}\right)\left(\lambda-S_{1}\right)\right. \\
& \left.\times \mathrm{A}_{1}(\lambda) F^{2}(\lambda)-I_{\mathrm{r}}(\lambda)\right\}
\end{aligned}
$$

where

$$
\begin{aligned}
I_{\mathrm{r}}(\hat{\lambda})= & 2 \lambda\left(\lambda-S_{\mathrm{r}}\right)\left\{\left(\lambda-S_{1}\right) B(\lambda) F(\lambda)-1\right\} \\
& \times A_{1}(\hat{\lambda}) F^{2}(\lambda)
\end{aligned}
$$

and

$$
B(\lambda)=\left(\lambda-S_{\mathrm{r}}\right)+\left(\lambda-S_{1}\right)+(\hat{\lambda}-1)
$$

On the other hand, the exponent of $\sigma_{\mathrm{r}}$ gives the average number of $\mathrm{RH} \alpha$-helices in the chain, since $\sigma_{\mathrm{r}}$ characterizes the initiation of the $\mathrm{RH} \alpha$ sequence

$$
\begin{aligned}
N_{\sigma_{\mathrm{r}}}= & \frac{\partial \ln Z_{N}}{\partial \ln \sigma_{\mathrm{r}}} \\
= & \frac{1}{Z_{N}} \sum_{\lambda} \lambda^{N-2}\left\{N\left(\lambda-S_{\mathrm{r}}\right)^{2}\left(\lambda-S_{\mathrm{l}}\right)\right. \\
& \left.\times A_{\mathrm{l}}(\lambda) F^{2}(\lambda)-J_{\mathrm{r}}(\lambda)\right\}
\end{aligned}
$$

where

$$
\begin{aligned}
J_{\mathrm{r}}(\lambda)= & \left(\lambda-S_{\mathrm{r}}\right)\left[2 \lambda\left(\lambda-S_{\mathrm{r}}\right)\left(\lambda-S_{1}\right) B(\lambda) F(\lambda)\right. \\
& \left.-\left\{2 \lambda^{2}-S_{\mathrm{r}}\left(\lambda+S_{1}\right)\right\}\right] A_{1}(\lambda) F^{2}(\lambda)
\end{aligned}
$$

Corresponding averages for the $\mathrm{LH} \alpha$-helix are

$$
\begin{aligned}
N_{S_{1}}= & \frac{1}{Z_{N}} \sum_{\lambda} \lambda^{N-1}\left\{N\left(\lambda-S_{\mathrm{r}}\right)\left(\lambda-S_{1}\right)\right. \\
& \left.\times A_{\mathrm{r}}(\hat{\lambda}) F^{2}(\hat{\lambda})-I_{1}(\hat{\lambda})\right\}
\end{aligned}
$$

and

$$
\begin{aligned}
N_{\sigma_{1}}= & \frac{1}{Z_{N}} \sum_{\lambda} \lambda^{N-2}\left\{N\left(\lambda-S_{\mathrm{r}}\right)\left(\lambda-S_{1}\right)^{2}\right. \\
& \left.\times A_{\mathrm{r}}(\lambda) F^{2}(\lambda)-J_{1}(\lambda)\right\}
\end{aligned}
$$

with

$$
\begin{aligned}
I_{1}(\lambda)= & 2 \lambda\left(\lambda-S_{1}\right)\left\{\left(\lambda-S_{\mathrm{r}}\right) B(\lambda) F(\lambda)-1\right\} \\
& \times A_{\mathrm{r}}(\lambda) F^{2}(\lambda)
\end{aligned}
$$

and

$$
\begin{aligned}
J_{1}(\lambda)= & \left(\lambda-S_{1}\right)\left[2 \lambda\left(\lambda-S_{\mathrm{r}}\right)\left(\lambda-S_{1}\right) B(\lambda) F(\lambda)\right. \\
& \left.-\left\{2 \lambda^{2}-S_{1}\left(\lambda+S_{\mathrm{r}}\right)\right\}\right] A_{\mathrm{r}}(\lambda) F^{2}(\lambda)
\end{aligned}
$$

Average fractions of helix units, $f_{\mathrm{h}}(\mathrm{h}=\mathrm{r}, 1)$, are then calculated as follows by considering again that the two chain-end residues do not adopt the $\alpha$-helical conformation

$$
f_{\mathrm{h}}=N_{S_{\mathrm{h}}} /(N-2)
$$

As the length $N$ increases, contribution of the leading eigenvalue $\lambda_{1}$ to $N_{S_{\mathrm{h}}}$ will become predominant over those of other two eigenvalues $\left(\lambda_{1}{ }^{N} \gg \lambda_{2}{ }^{N}, \lambda_{3}{ }^{N}\right)$. Thus, by introducing eq 15 (or 20 ) into 24 we have an asymptotic form of $f_{\mathrm{h}}$ at infinitely large $N$ as

$$
f_{\mathrm{h}}=A_{\mathrm{g}}\left(\lambda_{1}\right) F\left(\lambda_{1}\right) \quad(\mathrm{g} \neq \mathrm{h})
$$

Length Distribution Function and Moments of Helix

The simplified $3 \times 3$ matrix model enables us to deal with several more complex averages associated with the dimensional changes of a polypeptide chain in the sense inversion region. This advantage is taken below to calculate the length distribution and the higher order moments of helix.

The probability of occurrence of a $\mathrm{RH} \alpha$ sequence consisting of $v$ successive $r$ residues, $P_{\mathrm{r}}(v)$, may be given as ${ }^{19}$

$$
\begin{aligned}
P_{\mathrm{r}}(v)= & \frac{1}{Z_{N}}\left\{S_{\mathrm{r}}^{v}\left(\partial Z_{N-v+1} / \partial S_{\mathrm{r}}\right)\right. \\
& -2 S_{\mathrm{r}}{ }^{v+1}\left(\partial Z_{N-v} / \partial S_{\mathrm{r}}\right) \\
& +S_{\mathrm{r}}{ }^{v+2}\left(\partial Z_{N-v-1} / \partial S_{\mathrm{r}}\right)
\end{aligned}
$$


Here the first term derived from

$$
\begin{aligned}
& \frac{S_{\mathrm{r}}^{v}}{Z_{N}} \frac{\partial Z_{N-v+1}}{\partial S_{\mathrm{r}}} \\
& \quad=\frac{S_{\mathrm{r}}^{v}}{Z_{N}} \sum_{j=2}^{N-v}\left\{e_{1} W^{j-2}\left(\partial W / \partial S_{\mathrm{r}}\right) W^{N-j-v} e_{N}\right\}
\end{aligned}
$$

calculates the probability of finding of $\mathrm{RH} \alpha$ sequences of length $v$ and longer, while the second and third terms offset the overmeasured contributions from longer sequences. On introducing eq 14 into 26 we have

$$
\begin{aligned}
P_{\mathrm{r}}(v)= & \frac{\sigma_{\mathrm{r}} S_{\mathrm{r}}{ }^{v}}{Z_{N}} \sum_{\lambda} \lambda^{N-v-2}\left(\lambda-S_{\mathrm{r}}\right)\left(\lambda-S_{1}\right) \\
& \times\left[(N-v-1)\left(\lambda-S_{\mathrm{r}}\right)\left(\lambda-S_{1}\right)\right. \\
& +2 \lambda\left\{\left(\lambda-S_{\mathrm{r}}\right) A_{\mathrm{r}}(\lambda)\right. \\
& \left.\left.+\left(\lambda-S_{1}\right) A_{1}(\lambda)\right\} F(\lambda)\right] F^{2}(\lambda)
\end{aligned}
$$

A corresponding equation for a $\mathrm{LH} \alpha$ sequence, $P_{1}(v)$, is given by replacing $\sigma_{\mathrm{r}} S_{\mathrm{r}}{ }^{v}$ in eq 28 with $\sigma_{1} S_{1}{ }^{v}$. With these $P_{\mathrm{h}}(v)$ values, we can subsequently calculate the $n$th order moment of helix $M_{\mathrm{h}}{ }^{(n)}$ as

$$
M_{\mathrm{h}}{ }^{(n)}=\sum_{v=1}^{N-2} v^{n} P_{\mathrm{h}}(v)
$$

Note that the first moment $M_{\mathrm{h}}{ }^{(1)}$ satisfies the relation

$$
M_{\mathrm{h}}^{(1)}=\sum_{v=1}^{N-2} v P_{\mathrm{h}}(v)=N_{S_{\mathrm{h}}}
$$

hence reproduces $N_{S_{\mathrm{h}}}$ in eq 15 and 20, i.e., the average number of $\alpha$-helical residues per chain. In fact, introduction of eq 28 into 30 results in the same analytical expression for $N_{S_{\mathrm{r}}}$ given in eq 15.

\section{RESULTS AND DISCUSSION}

\section{Numerical Results}

To analyze the theory numerically, for a case of thermally induced transition, we define the equilibrium constant of the helix formation
$S_{\mathrm{h}}$ as a function of the absolute temperature $T$ :

$$
S_{\mathrm{h}}=\exp \left\{-\left(\Delta H_{\mathrm{h}} / R T\right)\left(1-T / T_{\mathrm{h}}\right)\right\}
$$

where $\Delta H_{\mathrm{h}}$ is the enthalpy change associated with the helix-coil transition occurring at $T_{\mathrm{h}}$ and $R$ is the gas constant. Transition parameters are taken from Part I so that the results of the two calculations can be compared under the same condition: $\Delta H_{\mathrm{r}}=-400 \mathrm{cal} \mathrm{mol}^{-1}$, $T_{\mathrm{r}}=310 \mathrm{~K}, \Delta H_{1}=-300 \mathrm{cal} \mathrm{mol}^{-1}, T_{1}=318 \mathrm{~K}$, and $\sigma_{\mathrm{r}}=\sigma_{1}=5 \times 10^{-5}$. The predominant chain conformation determined by this set of parameters shifts from $\mathrm{RH} \alpha$ to coil via an intermediate $\mathrm{LH} \alpha$ state as $T$ is increased. Figure 1 illustrates the temperature dependence of the average number of $\alpha$-helical residues $N_{S_{\mathrm{h}}}$ calculated at $N=400$. An initial increase in $N_{S_{1}}$ and an associated decrease in $N_{S_{\mathrm{r}}}$ represent the formation of $\mathrm{LH} \alpha$-helices proceeding at the expense of RH $\alpha$-helices. A subsequent decrease in $N_{S_{1}}$ corresponds to the melting of $\mathrm{LH}$ $\alpha$-helices into the random coil conformation. The total number of $\alpha$-helical residue $N_{S_{\mathrm{r}}}+N_{S_{1}}$ decreases only monotonically in the entire range of temperature, indicating that the sense inversion occurs without forming a respectably long coil sequence between RH and LH $\alpha$ helices. In other words, the propagation of the center of inversion along the chain should dominate the $\mathrm{RH} \alpha \rightarrow \mathrm{LH} \alpha$ transition.

The temperature dependence of the average number of $\alpha$-helical sequences $N_{\sigma_{\mathrm{h}}}$ is shown in Figure 2. $N_{\sigma_{\mathrm{r}}}$ in the $\mathrm{RH} \alpha$ region decreases asymptotically toward unity as $T$ is decreased. A value of $N_{\sigma_{\mathrm{r}}}=1.11$ at $T=250 \mathrm{~K}$ suggests, when compared with $N_{S_{\mathrm{r}}}=385$, that the chain at low temperatures includes a fairly long $\mathrm{RH}$ $\alpha$ sequence. As $T$ is increased to shift the conformer equilibrium to favor the LH $\alpha$ conformation, $N_{\sigma_{1}}$ begins to increase while $N_{\sigma_{\mathrm{r}}}$ decreases. Comparison of the $N_{\sigma_{1}}$ curve with the $N_{S_{1}}$ curve in Figure 1 indicates that the $\mathrm{LH}$ $\alpha$-helix grows in this region in both its average length and number. The $N_{\sigma_{1}}$ curve reaches its maximum at $T=310 \mathrm{~K}$. Correspondingly the total number of helices $N_{\sigma_{\mathrm{r}}}+N_{\sigma_{1}}$ runs up to 


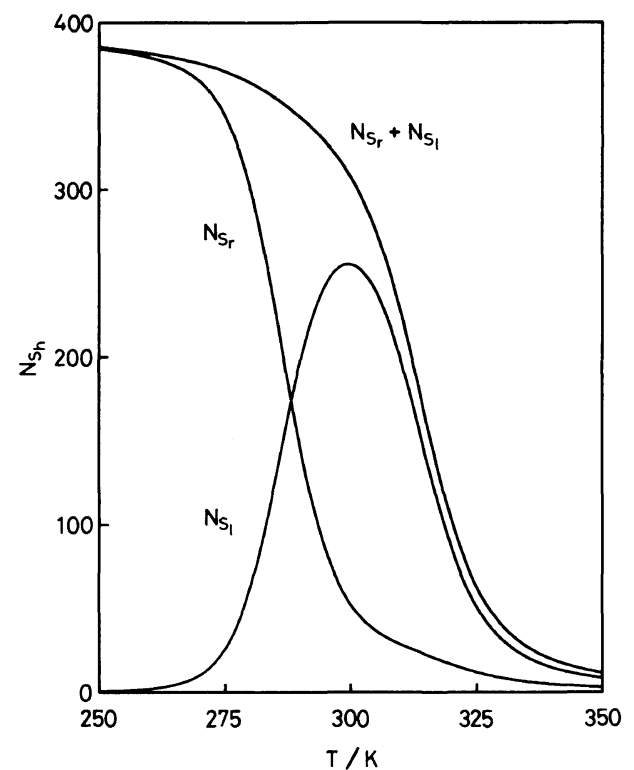

Figure 1. Temperature dependence of the average number of $\alpha$-helical residues $N_{S_{\mathrm{h}}}$ in a polypeptide chain of $N=400$ : Transition parameters assumed are $\Delta H_{\mathrm{r}}=$ $-400 \mathrm{cal} \mathrm{mol}^{-1}, \quad T_{\mathrm{r}}=310 \mathrm{~K}, \Delta H_{1}=-300 \mathrm{cal} \mathrm{mol}^{-1}$, $T_{1}=318 \mathrm{~K}$, and $\sigma_{\mathrm{r}}=\sigma_{1}=5 \times 10^{-5}$.

1.95 at the same temperature. This value is indicative of the coexistence of two $\alpha$-helices. Since $N_{\sigma_{1}}>N_{\sigma_{\mathrm{r}}}$, these coexisting helices are expected to consist mainly of $\mathrm{LH} \alpha-\mathrm{LH} \alpha$ and LH $\alpha-$ RH $\alpha$ pairs.

Further details of the sense inversion are seen in the helix-length distribution curves $P_{\mathrm{h}}(v)$ in Figure 3. For example, the RH $\alpha$-helix at $250 \mathrm{~K}$ has the average length of $N_{S_{\mathrm{r}}}=385$ (cf. Figure 1), the maximum $P_{\mathrm{r}}(v)$ value of 0.050 at $v=393$, and as much as $90 \%$ of $\mathrm{RH} \alpha$ residues are involved in sequences with $v=$ 350-398. The peak height of the $P_{\mathrm{r}}(v)$ curve decreases continuously with $T$, while the $P_{1}(v)$ curve, though much broader in width than $P_{\mathrm{r}}(v)$, begins to rise in the $\mathrm{LH} \alpha$ region above $280 \mathrm{~K}$. At $310 \mathrm{~K}$ where $N_{\sigma_{\mathrm{r}}}+N_{\sigma_{1}}$ is at its maximum in Figure 2, both $P_{\mathrm{r}}(v)$ and $P_{1}(v)$ curves exhibit almost linear decrease with $v$. The higher probabilities for the shorter helix sequences and $P_{1}(v)>P_{\mathrm{r}}(v)$ are congruous with the coexistence of LH $\alpha-\mathrm{LH} \alpha$ and $\mathrm{LH} \alpha-\mathrm{RH} \alpha$

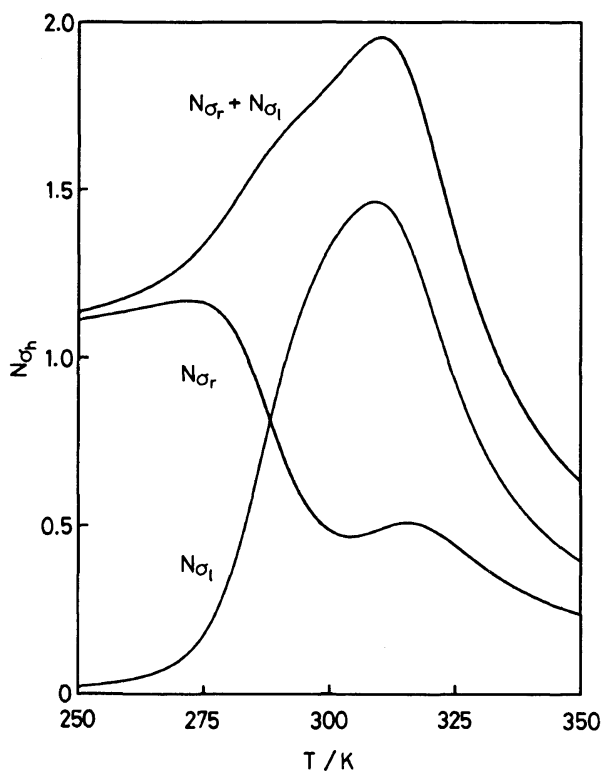

Figure 2. Temperature dependence of the average number of helix sequences $N_{\sigma_{\mathrm{h}}}$ at $N=400$.
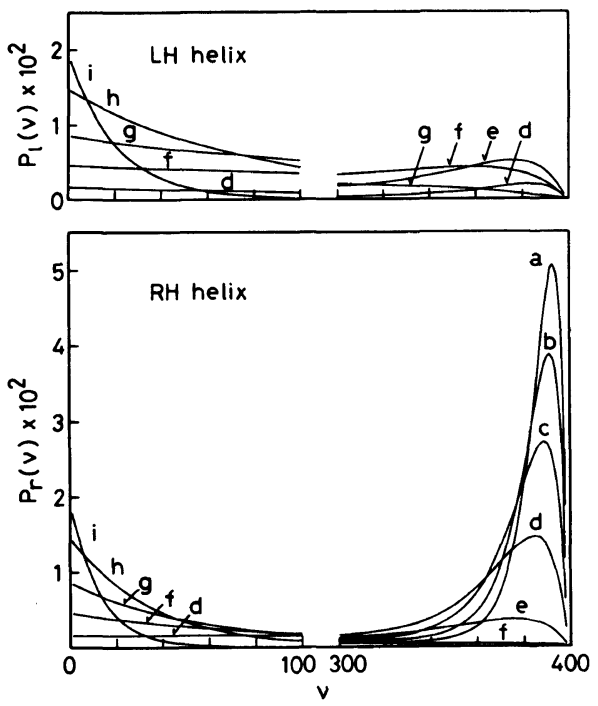

Figure 3. Helix-length distribution curves $P_{\mathrm{h}}(\mathrm{v})$ at (a) $T=250 \mathrm{~K}$, (b) $260 \mathrm{~K}$, (c) $270 \mathrm{~K}$, (d) $280 \mathrm{~K}$, (e) $290 \mathrm{~K}$, (f) $300 \mathrm{~K}$, (g) $310 \mathrm{~K}$, (h) $320 \mathrm{~K}$, and (i) $350 \mathrm{~K}$.

pairs suggested above.

Figure 4 shows the plots of $f_{\mathrm{r}}-f_{\mathrm{l}} v s . T$ at several different values of $N$. The $f_{\mathrm{r}}-f_{1}$ curves should correspond to the experimental transition curves determined by polarimetric meth- 


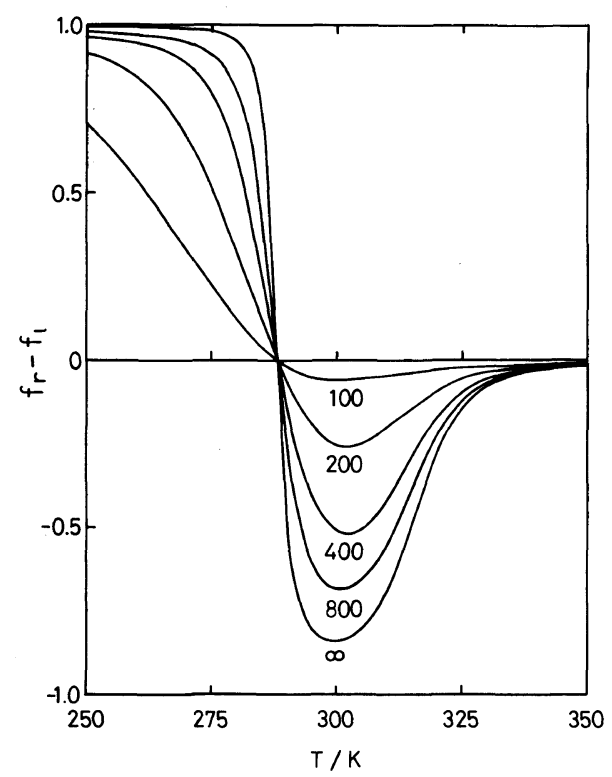

Figure 4. Curves of $f_{\mathrm{r}}-f_{1} v s . T$ at various values of $N$.

ods such as ORD and CD. The absolute $f_{\mathrm{r}}-f_{\mathrm{l}}$ value at any given temperature increases as $N$ is increased, i.e., the transition becomes sharper for a longer chain. However, the midpoint of the $\mathrm{RH} \alpha \rightarrow \mathrm{LH} \alpha$ transition remains at the same temperature independently of $N$. This critical temperature of the sense inversion, $T_{\text {inv }}$, is uniquely determined from eq 31 by equating $S_{\mathrm{r}}=S_{1}$ for a given set of $\Delta H_{\mathrm{h}}$ and $T_{\mathrm{h}}$ values ( $T_{\text {inv }}$ in the present example is $288.2 \mathrm{~K}$ ). Then, it should be straightforward to show, if $\sigma_{\mathrm{r}}=\sigma_{1}$, that $N_{S_{\mathrm{r}}}=N_{S_{1}}$ and $N_{\sigma_{\mathrm{r}}}=N_{\sigma_{1}}$ in eq $15-$ 23 , hence the chain at $T_{\text {inv }}$ includes the RH and LH $\alpha$-helical sequences having the same average conformation. The assumption that $\sigma_{\mathrm{r}}=\sigma_{1}$ may naturally be admitted for $\alpha$-helices coexisting in homopolypeptides. Even if $\sigma_{1} \neq \sigma_{1}$, since the $f_{\mathrm{r}}-f_{1}$ value at $T_{\mathrm{inv}}$ is less influenced by the difference in $\sigma_{\mathrm{h}}$ (note that $\sigma_{\mathrm{h}} \ll S_{\mathrm{h}}$ or unity), a shift of the crossover point will be confined within a very narrow range. Consequently, the convergence of the $f_{\mathrm{r}}-f_{1} v s$. $T$ curves at $T_{\text {inv }}$ appears as a general characteristic of the sense inversion phenomena occurring in homopolypeptides. This finding leads

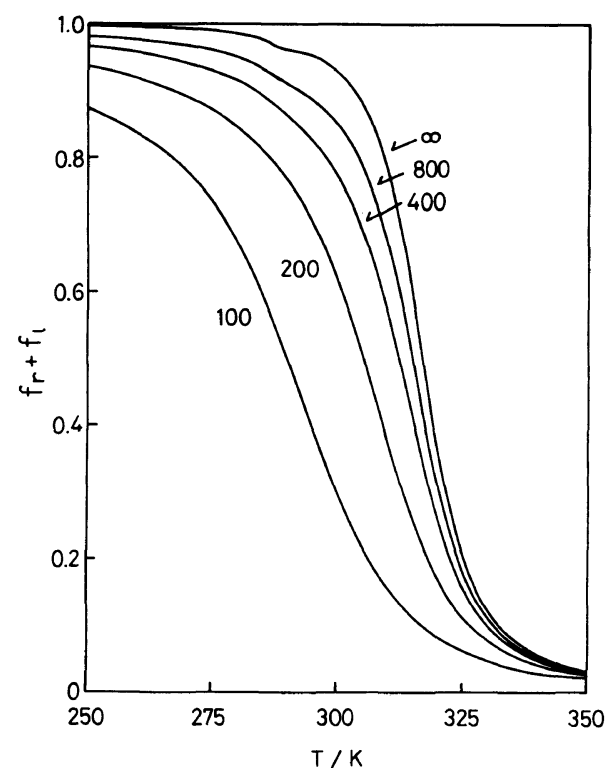

Figure 5. Curves of $f_{\mathrm{r}}+f_{1} v s . T$ at various values of $N$.

to the following suggestion concerning a method of estimation of the transition parameters: $\Delta H_{\mathrm{h}}$ and $T_{\mathrm{h}}$.

The $N$ dependence of the total helix fraction $f_{\mathrm{r}}+f_{1}$ is depicted in Figure 5. The $f_{\mathrm{r}}+f_{1}$ curve undergoes a sigmoidal decrease with $T$, and shifts continuously to the higher temperature region as $N$ is increased. Knowing in Figure 4 that $f_{\mathrm{r}}-f_{1}=0$ at the inversion midpoint, we obtain the $f_{\mathrm{r}}=f_{1}$ value from Figure 5 as a function of $N$. Under a condition that $S_{\mathrm{r}}=S_{\mathrm{l}}$ $(=S)$ and $\sigma_{\mathrm{r}}=\sigma_{1}(=\sigma / 2)$ all our equations are reduced to those given by the Zimm-Bragg theory ${ }^{14}$ for a simple helix-coil transition: Mathematics necessary for evaluating $S$ and $\sigma$ from the $f$ vs. $N$ curve can be found in the theories subsequently developed. ${ }^{21-23}$ Having had the $S$ and $\sigma$ values established at $T_{\text {inv }}$, we can then optimize the $\Delta H_{\mathrm{h}}$ and $T_{\mathrm{h}}$ parameters by analyzing either the $f_{\mathrm{r}}-f_{1}$ curves in Figure 4 or the $f_{\mathrm{r}}+f_{1}$ curves in Figure 5 as a function of temperature (via eq 31). It may be noted that the number of independent parameters to be optimized decreases to two (for the simplest case where $\sigma_{\mathrm{r}}=\sigma_{1}$ ) because of the aforemen- 


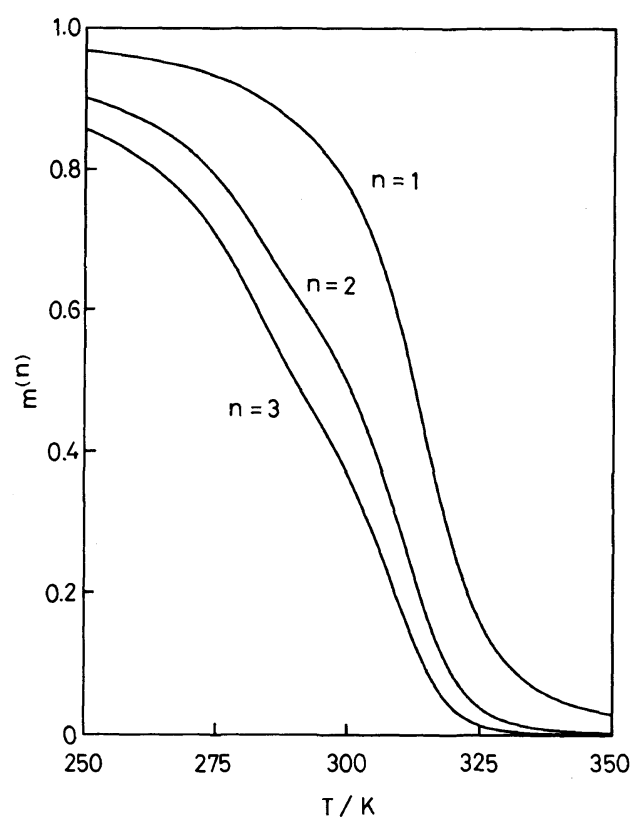

Figure 6. Curves of $m^{(n)}=\left(M_{\mathrm{r}}^{(n)}+M_{\mathrm{l}}^{(n)}\right) /(N-2)^{n}$ $(n=1-3)$ at $N=400$.

tioned peculiarity of the $\mathrm{RH} \alpha \rightarrow \mathrm{LH} \alpha$ sense inversion.

The above analyses may alternatively be carried out by using the higher moments of helix. Figure 6 illustrates how the first three moments, $m^{(n)}=\left(M_{\mathrm{r}}^{(n)}+M_{1}^{(n)}\right) /(N-2)^{n}(n=1-$ $3)$, vary with temperature in the sense inversion region. The higher moments reflect the mode of helix-length distribution more sensitively, hence can reveal subtle changes in the average chain dimension. For example, the $m^{(2)}$ and $m^{(3)}$ curves show a shallow dent in the sense inversion region, whilst no such a change is seen when the first moment $m^{(1)}\left(\equiv f_{\mathrm{r}}+f_{1}\right)$ alone is examined. Further refinement of the theory to include the effects of spatial distribution and flexibility of $\alpha$-helices coexisting in a chain is certainly possible, and will be discussed in detail for the case of the electric birefringence of PCBL in the helix-coil transition region. ${ }^{9}$

\section{Comparison with Previous Calculations}

To compare the numerical results obtained in the present study with those in Part I, we evaluate the root-mean-square (rms) errors between the two sets of results:

$$
\sqrt{(1 / n) \sum_{i=1}^{n}\left(x_{1}-x_{2}\right)^{2}}
$$

where $x_{1}$ and $x_{2}$ are the values from previous and present calculations, respectively, and $n=$ 101 is the number of data points between $T=$ $250 \mathrm{~K}$ and $300 \mathrm{~K}$. The rms error for $N_{S_{\mathrm{r}}}$ at $N=400$ is calculated to be 0.864 and that for $N_{S_{1}}$ to be 0.955 . Both values are satisfactorily small compared with the total number of residues $N=400$, hence differences between the two sets of results cannot be distinguished in the curves in Figure 1. Similar agreement is also observed for $N_{S_{\mathrm{h}}}$ at different values of $N$ and for other averages.

The primary difference between the previous and present theories lies in the degree of approximation concerning the helix stabilization effects due to hydrogen-bond interactions. In Part I, such interactions between four consecutive residues are explicitly expressed in the $9 \times 9$ statistical-weight matrix. Among five non-zero eigenvalues of this matrix, only three have the values of the order of $S_{\mathrm{h}}$ or unity, while the remaining two are in the order of $\sqrt{\sigma_{\mathrm{h}}} \simeq 10^{-2}$. Obviously, contributions of the latter two eigenvalues to the partition function are smaller than those of the three leading eigenvalues. The present theory, based on the $3 \times 3$ statistical-weight matrix, reproduces these three major eigenvalues. Although short, unfavorable helix sequences (chc and chhc) are not excluded in this nearest-neighbor interaction model, their influences to the final numerical results are found to be only trivial.

\section{Implication with Experiments}

As we have pointed out above, analyses of the sense inversion mechanism should require not only the $f_{\mathrm{r}}-f_{1}$ value available from ORD and $\mathrm{CD}$ experiments, but also the $f_{\mathrm{r}}+f_{\mathrm{l}}$ value or its equivalent that reflects the average dimension of helix. The most straightforward 
method for estimating $f_{\mathrm{r}}+f_{1}$ should be a ultraviolet absorption measurement. There are, however, several other feasible approaches that can discern the details of the average chain dimension. They include the analyses of the higher order moments of helix rather than the first moment. Especially, the mean-square dipole moment and the Kerr constant, which respectively reflect the second and third moments of helix (see Figure 6), are of our interest. Omura et al. ${ }^{12}$ have analyzed the dipole moment of PCBL in the helix-coil transition region, resulting in a $\sigma$ value coincident with that obtained from ORD measurements. More recently, we have shown for the same polypeptide that the changes in its electric birefringence can similarly be reproduced from ORD data. ${ }^{9}$ These studies suggest that the average dimension of helix is experimentally measurable, hence can be collaborated with ORD and CD data in the analyses of the sense inversion mechanism. With the new theory it is even possible to calculate the meansquare radius of gyration, which may be compared with the informations from light scattering measurements. The Nagai theory of the helix-coil transition, ${ }^{19}$ in which helix sequences (rigid rods) and coil sequences (random flight chains) are assumed to be alternately connected by flexible joints, can be expanded to apply to the sense inversion case. Determination and analyses of these quantities are beyond the scope of this study; nevertheless, it should just be noted that the $f_{\mathrm{r}}-f_{1}$ curves in Figure 4 can reproduce the ORD data reported for PPELA, ${ }^{2}$ thus confirming the validity of our theory.

In summary, the theory of the helix-sense inversion in polypeptides, simplified within a framework of the three-state, nearest-neighbor interaction model, improves its applicability and provides a mathematical basis for analyzing the experimental observations. The new theory is shown to be particularly advantageous in calculating several complex aver- ages associated with dimensional changes of polypeptides. Analyses of the calculated transition curves reveal general characteristics of the sense inversion phenomena and suggest a procedure for evaluating the transition parameters. The proposed method will be subjected to a quantitative comparison with experiments in subsequent studies.

\section{REFERENCES}

1. H. Toriumi, Macromolecules, 17, 1599 (1984).

2. H. Toriumi, N. Saso, Y. Yasumoto, S. Sasaki, and I. Uematsu, Polym. J., 12, 977 (1979).

3. E. M. Bradbury, B. G. Carpenter, and H. Goldman, Biopolymers, 6, 837 (1968).

4. E. M. Bradbury, B. G. Carpenter, and R. M. Stephens, Biopolymers, 6, 905 (1968).

5. E. M. Bradbury, B. G. Carpenter, C. C. Robinson, and H. Goldman, Macromolecules, 4, 557 (1971).

6. M. Hashimoto and J. Aritomi, Bull. Chem. Soc. Jpn., 39, 2707 (1966).

7. M. Hashimoto and S. Arakawa, Bull. Chem. Soc. Jpn., 40, 1698 (1967).

8. E. H. Erenrich, R. H. Andreatta, and H. A. Scheraga, J. Am. Chem. Soc., 92, 1116 (1970).

9. H. Toriumi, H. Sugisawa, and H. Watanabe, in preparation.

10. M. Matsuoka, T. Norisuye, A. Teramoto, and H. Fujita, Biopolymers, 12, 1515 (1973).

11. K. Nakamoto, H. Suga, S. Seki, A. Teramoto, T. Norisuye, and H. Fujita, Macromolecules, 7, 784 (1974).

12. I. Omura, A. Teramoto, and H. Fujita, Macromolecules, 8, 284 (1975).

13. N. Nishioka, A. Maekawa, and A. Teramoto, Biopolymers, 17, 665 (1978).

14. B.H. Zimm and J. K. Bragg, J. Chem. Phys., 31, 526 (1959).

15. R. A. Scott, Biopolymers, 5, 931 (1967).

16. T. Ooi, R. A. Scott, G. Vanderkooi, and H. A. Scheraga, J. Chem. Phys., 46, 4410 (1967).

17. J. F. Yan, G. Vanderkooi, and H. A. Scheraga, $J$. Chem. Phys., 49, 2713 (1968).

18. K. Nagai, J. Phys. Soc. Jpn., 15, 407 (1960).

19. K. Nagai, J. Chem. Phys., 34, 887 (1961).

20. A. Teramoto and H. Fujita, J. Macromol. Sci., Rev. Macromol. Chem., C15, 165 (1976).

21. B. H. Zimm, P. Doty, and K. Iso, Proc. Natl. Acad. Sci. U.S.A., 45, 1601 (1959).

22. J. Applequist, J. Chem. Phys., 38, 934 (1963).

23. K. Okita, A. Teramoto, and H. Fujita, Biopolymers, 9, 717 (1970). 